\title{
Aksak Patterns and Entrained Interaction in Transylvanian Village Music
}

\author{
MARTIN CLAYTON \\ Durham University
}

\begin{abstract}
In this response to Filippo Bonini Baraldi, Emmanuel Bigand and Thierry Pozzo's article 'Measuring aksak rhythm and synchronization in Transylvanian village music by using motion capture', I present supplementary analyses of (a) the ratio between Short and Long beats, and (b) the entrainment between the two musicians in the motion capture recordings. The main findings reported are: the mean S:L ratio is close to $1: \sqrt{ } 2$, although there is some evidence for the role of $2: 3$ as an attractor ratio; the distribution of S:L ratios and other measures vary depending on whether the period is taken as $\mathrm{S}+\mathrm{L}$ or $\mathrm{L}+\mathrm{S}$; and the $\mathrm{S}: \mathrm{L}$ ratio varies with tempo. Since the viola part is much less variable than the violin part, the former should be taken as a reference; the violinist tends to play ahead of the beat articulated by the violist, significantly so except for the Short beat in one recording (Duo 14), in which the musicians exhibit a form of soft entrainment, alternating between small and large phase differences.
\end{abstract}

Submitted 2015 May 29; accepted 2015 June 10.

KEYWORDS: aksak, nonisochronous rhythm, entrainment

THIS valuable target article brings a number of important themes in music theory, empirical musicology and ethnomusicology into a fruitful juxtaposition. From ethnomusicology comes the theory of aksak rhythm, which has been pursued largely in Francophone scholarship and often ignored elsewhere. From music theory, the authors bring aksak's analogue 'nonisochronous metre', and its language of Short and Long beat classes. From empirical musicology, the language of 'asynchronies' and the techniques of motion capture are added to the mix; from psychology, the framework of interpersonal coordination. As the authors suggest, the result is a significant contribution to the emergent field of 'non-ethnocentric analytical musicology'.

In duet performances (on violin and viola) of Transylvanian village music, Bonini-Baraldi et al explore how empirical methods such as onset detection and motion capture can illuminate both the details of an SLSL aksak rhythm, and the nature of coordination between the two parts. In brief, we learn that the performed rhythms generally fall somewhere between the 2:3 and 3:4 ratios, albeit with considerable variation, and that the viola player is more often behind than ahead of the violinist as the two combine to produce a marked flexibility in the perceived beat. The data the authors provide on durations, tempi and S:L ratios are most welcome, since aksak is an area in which we are desperately short of empirical studies. In the discussion on coordination between the two musicians, Bonini Baraldi's qualitative interpretation, based on his ethnographic experience, sheds considerable light where quantitative data alone might bring only confusion. The numbers are a trace of an ongoing interaction between two musicians, in which leadership roles are assumed and may be contested, where individual musicians either take the opportunity to show their individual style or else content themselves with a supporting role. As an ethnomusicologist the realization that ethnography has something unique to contribute here is reassuring, if unsurprising; nonetheless, as an empirical musicologist I wonder if the data could in fact say more about both the rhythmic structure and the dynamics of interaction.

In this response, therefore, I concentrate on further analysis of the original timing data with the intention of further testing the authors' interpretations. The contribution is in two sections: the first considers aspects of the ratio between $\mathrm{S}$ and $\mathrm{L}$ beat classes in this repertoire, while the second applies entrainment analysis to the original asynchrony data. 


\section{ASPECTS OF THE S:L RATIO}

The target article adds to a small but growing body of literature on non-isochronous beats and subdivisions. Much of this literature concerns 'swing ratios' in jazz (Benadon 2006) and analogous phenomena in Scandinavian dance music (Kvifte 2007) and African musical traditions (Jankowsky 2013, Polak and London 2014): what these examples have in common is an uneven subdivision of the beat in which the ratio between Long and Short elements lies somewhere between 1:1 and 1:2. Empirical studies show a range of ratios, many of which cannot be considered close to integer ratios such as 2:3 or to 3:4. It has become clear, after some debate, that everything cannot be reduced to a fast isochronous pulse.[1]

With longer time intervals - when beats are considered, rather than subdivisions - the music psychology literature since Fraisse focuses on the preference for a 1:2 ratio between Shorts and Longs. Fraisse suggested that there is a boundary between Shorts and Longs at around 400-600 msec, the so-called 'indifference interval' (1978). Later work complicates this picture somewhat, but remains focused on attractor ratios on or around 1:2 and time intervals of this magnitude, and so does not directly tackle either the possibility of $2: 3$ or $3: 4$ attractor ratios or the rather longer durations encountered in the current example (Shorts around $1000 \mathrm{msec}$ ).[2] Laboratory studies which explicitly include 2:3 ratios include Collier and Wright (1995) and Semjen and Ivry (2001), neither of which however address the stability of this proportion in a sustained way. A comparison of these studies, in fact, reveals a lack of consensus on how this ratio should be treated: Collier and Wright categorise 2:3 with other 'simple' ratios such as 1:2 which are taken to be relatively stable under tempo changes, whereas Semjen and Ivry write the same ratio as $1.5: 1$, describing it as a non-integer ratio and appearing to make the opposite assumption.

Little empirical work has been done on S:L ratios in performed aksak rhythms prior to the current article. As Bonini Baraldi et al note, Bouët's work throws any assumption of a preferred 2:3 ratio in aksak into doubt (1997). In fact, Singer had pointed out forty years ago that in Macedonian dance tunes this ratio is often far from 2:3 (1974: 386), while Kvifte's more recent analysis of note pairs in a Norwegian springar tune, which seem quite evenly spread over a broad range, is a simple demonstration of the fact that interval ratios do not necessarily cluster around small integer ratios (2007: 71). Bouët argues strongly against Arom's claim that an isochronous subdivision must underpin aksak patterns,[3] proposing instead a 'bichronous' interpretation in which Short and Long are distinct categories rather than multiples of a minimal unit. The majority view here therefore accords with current thinking in music theory and empirical musicology. While there are doubtless 'aksak' rhythms performed with a clearly articulated isochronous pulse (Shorts divided into 2, Longs into 3), the present case is not an example of this, and lends further support to the 'bichronous' interpretation. The question remains, in such a case do either 2:3 or 3:4 act as attractor ratios or do they not? The following sections explore Bonini Baraldi et al's data further with a view to exploring this and related questions.

\section{1a. What is the $\mathrm{S}: \mathrm{L}$ ratio?}

This question actually embodies a number of questions:

- Do the data further support the contention that $\mathrm{S}$ and $\mathrm{L}$ are simply two distinct beat (interval) categories, as opposed to multiples of some basic durational unit?

- If the former, do the 2:3 and 3:4 ratios have any significance, or are the instantaneous ratios simply spread out over a range of values between, but distant from, both 1:1 and 1:2? [4]

- If we presume an intention to maintain a ratio as far as possible from both 1:1 and 1:2, with no regular subdivision and with no other constraints, we might expect the resulting ratios to tend towards the geometric mean of these ratios, $1: \sqrt{2}$, or around 0.707.[5] Is there any evidence for this in practice?

- Is there any difference in the variability of Ss and Ls (which might for instance indicate constraints on absolute intervals)?

- Further, does the implicit grouping of beats identified by musicologists as $\mathrm{S}+\mathrm{L}$ (rather than $\mathrm{L}+\mathrm{S}$ ) make any difference to the distribution of the ratios (which might inform us about the nature of the motor timing control, see below)?

Using Bonini Baraldi et al's original motion capture data for the beat onsets for Duos 12-14 and 18-22, Table 1 summarizes the $\mathrm{S}: \mathrm{L}$ ratios for each recording, as well as means and coefficients of variation (CV) for the $\mathrm{S}$ and $\mathrm{L}$ beats, the $\mathrm{S}+\mathrm{L}$ groups (calculated both ways, i.e. Short and the following Long, Long with 
the following Short) and the tempo (using the authors' method of taking the $\mathrm{S}$ beat as the reference). The $\mathrm{CV}$ measures suggest there is little difference between the variability of $\mathrm{S}$ and $\mathrm{L}$ beats ( $\mathrm{L}$ being on average slightly more variable than $S$ ). The variability of the period was calculated both ways round $(S+L, L+S)$ to allow comparison with Semjen and Ivry's finding that variability was higher (in an analogous tapping task) when the cycle was measured with the shorter of two intervals first (2001). This is also the case with the current data, although the margin is small (CVs of $4.6 \%$ vs $4.18 \%$ ) and the pattern inconsistent. Semjen and Ivry argue that a difference in variability indicates that the timing is controlled by a hierarchical rather than a serial timer (2001: 254). If the difference were considered to be significant in this case, it could indicate that the timing of the period is controlled as an $\mathrm{L}+\mathrm{S}$ group rather than an $\mathrm{S}+\mathrm{L}$ group. The difference here is too slight to offer strong evidence of this, but this is a factor that may be worth investigating in future studies.

There is more diversity in the variability of the S:L ratios, with Duos 12 and 18 being less variable and Duo 19 more variable (although the latter effect disappears when the ratios are calculated with the $\mathrm{L}$ interval first, dropping from 9.8 to $4.6 \%$ ). It's not obvious to this novice listener why this might be the case.

Table 1. Mean and CV figures for Short and Long beats, $\mathrm{S}+\mathrm{L}$ and $\mathrm{L}+\mathrm{S}$ periods, Tempo and $\mathrm{S}: \mathrm{L}$ ratios for all Duo recordings.[6]

\begin{tabular}{|l|l|l|l|l|l|l|l|l|l|l|l|l|}
\hline & \multicolumn{10}{l|}{ MEANS } & \multicolumn{1}{l|}{ CVs } \\
\hline & S (s) & L (s) & $\begin{array}{l}\text { S+L } \\
\text { (s) }\end{array}$ & $\begin{array}{l}\text { L+S } \\
\text { (s) }\end{array}$ & $\begin{array}{l}\text { Tempo } \\
(\mathrm{bpm})\end{array}$ & $\begin{array}{l}\text { Ratio } \\
\text { S:L }\end{array}$ & S & L & S+L & L+S & Tempo & $\begin{array}{l}\text { Ratio } \\
\text { S:L }\end{array}$ \\
\hline Duo 12 & 1.10 & 1.58 & 2.67 & 2.69 & 56.2 & 0.696 & $4.40 \%$ & $4.56 \%$ & $4.06 \%$ & $2.97 \%$ & $4.14 \%$ & $3.91 \%$ \\
\hline Duo 13 & 1.10 & 1.58 & 2.67 & 2.66 & 56.2 & 0.696 & $4.90 \%$ & $4.27 \%$ & $3.78 \%$ & $3.38 \%$ & $3.76 \%$ & $5.08 \%$ \\
\hline Duo 14 & 1.16 & 1.65 & 2.81 & 2.80 & 53.6 & 0.700 & $5.56 \%$ & $5.78 \%$ & $4.89 \%$ & $4.64 \%$ & $4.91 \%$ & $6.44 \%$ \\
\hline Duo 18 & 1.11 & 1.51 & 2.62 & 2.62 & 57.4 & 0.735 & $6.60 \%$ & $5.52 \%$ & $5.68 \%$ & $4.96 \%$ & $5.73 \%$ & $3.88 \%$ \\
\hline Duo 19 & 1.18 & 1.74 & 2.92 & 2.90 & 51.4 & 0.679 & $5.12 \%$ & $7.47 \%$ & $4.72 \%$ & $6.21 \%$ & $4.90 \%$ & $9.76 \%$ \\
\hline Duo 20 & 1.10 & 1.60 & 2.70 & 2.70 & 55.7 & 0.689 & $3.85 \%$ & $5.84 \%$ & $3.66 \%$ & $4.07 \%$ & $3.63 \%$ & $7.13 \%$ \\
\hline Duo 21 & 1.04 & 1.52 & 2.57 & 2.57 & 58.7 & 0.687 & $5.72 \%$ & $6.55 \%$ & $5.66 \%$ & $3.50 \%$ & $6.10 \%$ & $5.24 \%$ \\
\hline Duo 22 & 1.13 & 1.56 & 2.69 & 2.68 & 55.9 & 0.729 & $5.06 \%$ & $5.11 \%$ & $4.38 \%$ & $3.73 \%$ & $4.35 \%$ & $5.26 \%$ \\
\hline Average & 1.11 & 1.59 & 2.71 & 2.70 & 55.6 & 0.701 & $5.15 \%$ & $5.64 \%$ & $4.60 \%$ & $4.18 \%$ & $4.69 \%$ & $5.84 \%$ \\
\hline
\end{tabular}

The mean S:L ratios, as we know from the target article, all lie in the range between 2:3 (0.667) and 3:4 (0.75), although the instantaneous figures often exceed those limits. It is also true, however, that the overall average of 0.701 is closer to the $1: \sqrt{2}$ ratio of 0.707 than it is to either $2: 3$ or $3: 4$. The summary data do not definitively answer the question: do the ratios tend towards either $2: 3$ or $3: 4$, or do they follow a normal distribution centered on a mean of 0.707 ? A histogram of all instantaneous S:L ratio calculations shows a pattern close to a normal distribution around 0.7 (Figure 1a), with a peak just above this figure.[7] It also shows a second peak close to 0.667 (2:3), but no peak around 0.75 (3:4). A second view of the same data is obtained by calculating the ratio between each $\mathrm{L}$ and the following $\mathrm{S}$ (Figure 1b). The overall mean is (inevitably) almost the same whichever way round the ratio is calculated. Interestingly, however, Figure $1 \mathrm{~b}$ shows that in this case the highest peak in the distribution is in fact close to 0.667 (2:3). This could indicate that the influence of an $\mathrm{L}$ beat on the following $\mathrm{S}$ is stronger than that of an $\mathrm{S}$ beat on the following $\mathrm{L}$.

The histograms are consistent with the following interpretation: that in general, the $\mathrm{S}$ and $\mathrm{L}$ are distinct beat classes; $\mathrm{L}$ is always longer than $\mathrm{S}$ but not so long that it can be easily divided into $2 \mathrm{x}$. These beat classes are not consistently subdivided into $2+3$ pulses or into $3+4$ pulses, but they are intermittently subdivided, and this may explain the fact that the distribution differs a little from a normal distribution curve. 

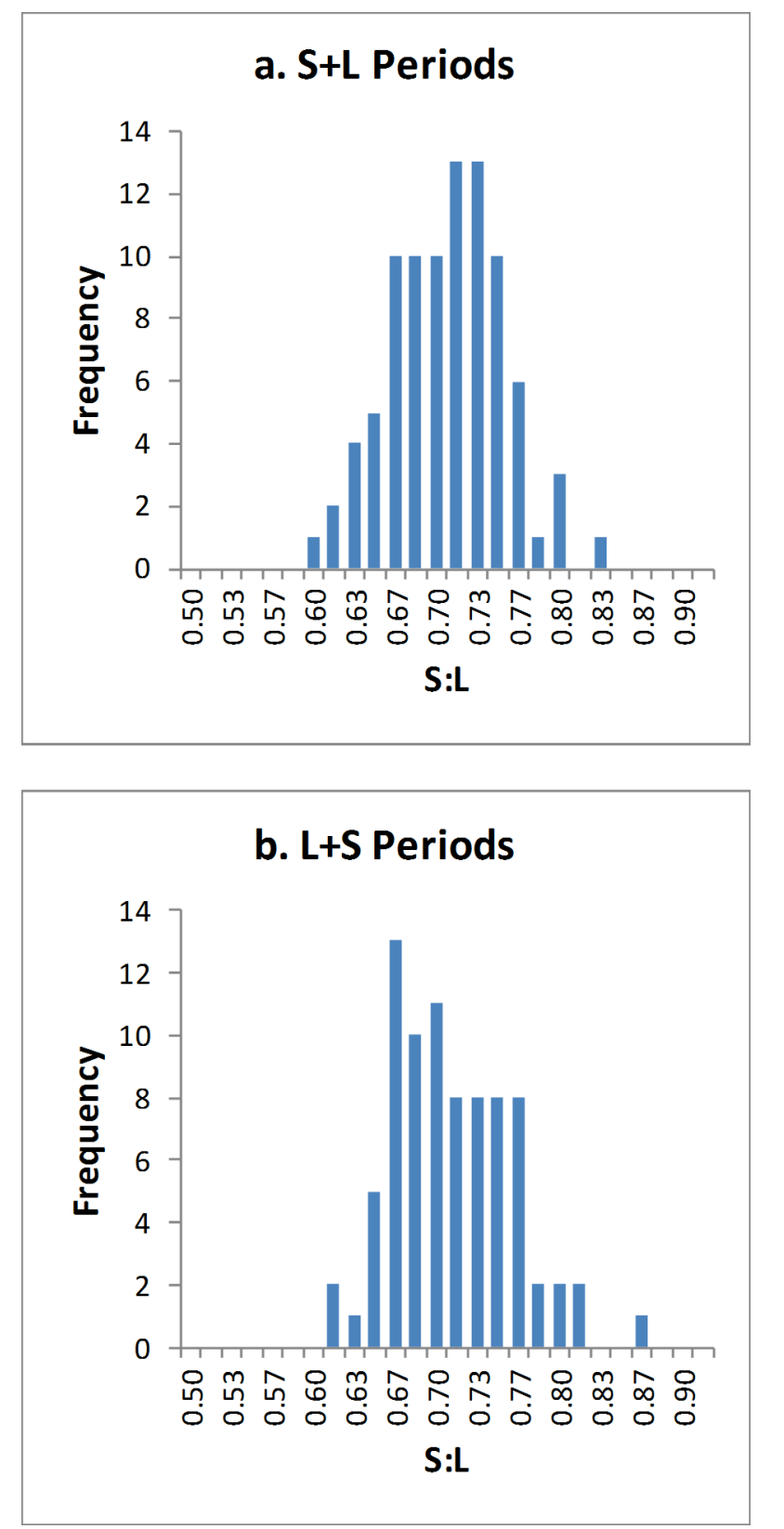

Figure 1. S:L ratio distributions. (a) calculated for $\mathrm{S}+\mathrm{L}$ periods, (b) calculated for $\mathrm{L}+\mathrm{S}$ periods.

What is the significance in the apparently different distributions depending on which way round the ratios are calculated? One possibility is that subdivision of the L group sometimes leads to a tacit subdivision of the following $\mathrm{S}$ into 2 parts, resulting in a ratio closer to 2:3. In support of this argument, the violinist's subdivision of the $\mathrm{S}$ beat is very varied: as shown in the transcription in Bonini Baraldi et al's Figure 2, he may use 1, 2 or 3 note onsets, with the perceived subdivision shifting between duple and triple. In contrast, the $\mathrm{L}$ is sometimes subdivided by the violinist into one long note followed by an ornamented figure functioning as an anacrusis. 


\section{1b. Is there any difference in the variability of the viola and violin parts?}

Timing data for the viola and violin onsets for Duos 12-14 (three versions of the same tune) are summarized in Table 2. They show a consistent pattern: the CV measures for the violin are in every case higher than those for the viola, while the S:L ratios are lower, slightly in Duos 12 and 13 but significantly so in Duo 14, where they go below 0.5 (1:2). It is clear that in these recordings at least, the viola maintains a relatively steady tempo (slightly slower in Duo 14 ) and a consistent S:L ratio of about 0.7 . The violin part is far more variable - or flexible - and the difference between $\mathrm{S}$ and $\mathrm{L}$ tends to be exaggerated, with the $\mathrm{L}$ often more than twice as long as the adjacent S. What this throws into some doubt is Bonini Baraldi et al's tentative interpretation that the violist waits for the violinist [p.281]: were this the case one would expect the viola part to be at least as variable as the violin part. It seems more reasonable to regard the viola part, being less variable and therefore more predictable, as a referent around which the violin part varies.

Table 2. Means and CVs for S+L period (sec), Tempo (bpm), and S:L ratio calculated for $\mathrm{S}+\mathrm{L}$ periods and $\mathrm{L}+\mathrm{S}$ periods (Inv Ratio). Separate figures for viola and violin (left to right) and for Duos 12, 13 and 14 (top to bottom).

\begin{tabular}{|l|l|l|l|l|l|l|l|l|}
\hline & \multicolumn{4}{|l|}{ VIOLA } & \multicolumn{2}{l|}{ VIOLIN } \\
\hline Duo 12 & S+L & Tempo & Ratio S:L & Inv Ratio S:L & S+L & Tempo & Ratio S:L & Inv Ratio S:L \\
\hline Mean & 2.67 & 56.19 & 0.696 & 0.699 & 2.69 & 56.42 & 0.578 & 0.607 \\
\hline CV & $4.06 \%$ & $4.14 \%$ & $3.91 \%$ & $6.68 \%$ & $12.29 \%$ & $11.94 \%$ & $22.38 \%$ & $27.54 \%$ \\
\hline
\end{tabular}

\begin{tabular}{|l|l|l|l|l|l|l|l|l|}
\hline Duo 13 & S+L & Tempo & Ratio S:L & Inv Ratio S:L & S $+\mathrm{L}$ & Tempo & Ratio S:L & Inv Ratio S:L \\
\hline Mean & 2.67 & 56.33 & 0.692 & 0.697 & 2.54 & 59.78 & 0.585 & 0.692 \\
\hline CV & $3.88 \%$ & $3.84 \%$ & $4.93 \%$ & $6.06 \%$ & $11.84 \%$ & $11.81 \%$ & $17.85 \%$ & $32.12 \%$ \\
\hline
\end{tabular}

\begin{tabular}{|l|l|l|l|l|l|l|l|l|}
\hline Duo 14 & S+L & Tempo & Ratio S:L & Inv Ratio S:L & S+L & Tempo & Ratio S:L & Inv Ratio S:L \\
\hline Mean & 2.81 & 53.56 & 0.700 & 0.696 & 2.83 & 53.44 & 0.473 & 0.482 \\
\hline CV & $4.89 \%$ & $4.91 \%$ & $6.44 \%$ & $6.39 \%$ & $8.92 \%$ & $9.75 \%$ & $40.75 \%$ & $40.50 \%$ \\
\hline
\end{tabular}

\section{1c. Is there any relationship between the $S: L$ ratio and tempo?}

There is little in the current literature that speaks to possible relationships between S:L ratios and tempo. However, Jankowsky's study of Tunisian Stambeli ritual music investigates the ratios between elements in three- and four-element non-isochronous rhythmic patterns as performances accelerate (2013). In some cases there seems to be a clear assimilation of the intervals as tempo increases (i.e. the $\mathrm{S}: \mathrm{L}$ ratio flattens out, tending towards 1 as tempo increases).[8] In the current case, tempo does not change significantly over the course of any recording, or vary greatly between examples - average tempi all lie between 51.4 and $58.7 \mathrm{bpm}$. Is there nonetheless any correlation between tempo and S:L ratio? In Figure 2, the S:L ratio is plotted against tempo for each data point of the Duo recordings. The trend line shows this ratio increasing with tempo: as Jankowsky found with larger accelerations in Stambeli, as tempo increases so does the $\mathrm{S}: \mathrm{L}$ ratio (Pearson $r=0.232, \mathrm{p}<.05$ ). Interestingly, if we use the inverse ratios ( $\mathrm{L}+\mathrm{S}$ groupings), the correlation is higher $(r=0.665, \mathrm{p}<.01)$ : note the increased slope of the trendline in Figure $2 \mathrm{~b}$. 

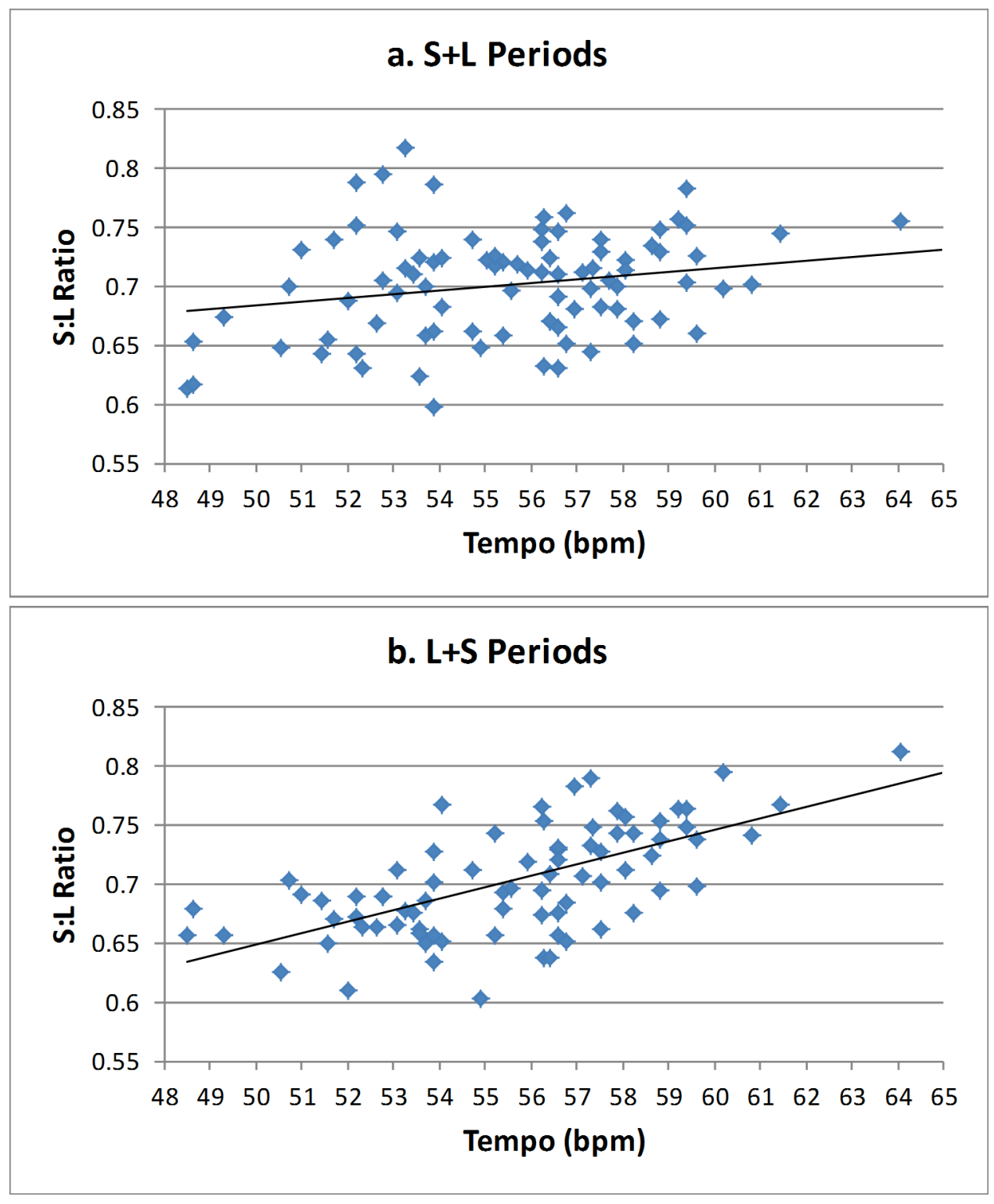

Figure 2. S:L Ratios plotted against Tempo, with linear trend lines. (a) for $\mathrm{S}+\mathrm{L}$ periods, (b) for $\mathrm{L}+\mathrm{S}$ periods.

\section{ENTRAINMENT ANALYSIS}

The second set of questions I wish to address here asks, would an entrainment analysis of the relationship between viola and violin parts in Duos 12-14 reveal details that do not show up in Bonini Baraldi et al's asynchrony analysis? The authors describe the relationship between violin and viola onsets in terms of absolute asynchronies: raw asynchrony data, however, are not very helpful in interpreting the coordination between musicians, which can be studied more effectively by calculating relative phase relationships (Clayton, Sager and Will 2005). If Bonini Baraldi et al's onset data are recalculated as phase relationships and their mean vectors computed, the overall picture is illustrated in Table 3. On the main S and L beats, the violin onsets are found on average to fall between 22 and $34^{\circ}$ before the corresponding viola onsets (326-338 , roughly $1 / 16$ th to $1 / 10$ th of the $\mathrm{S}+\mathrm{L}$ period). Mean vector lengths (a measure of how tightly coordinated the two parts are) are all over 0.9: the lowest figure, 0.906, is for the violin in relation to the viola in Duo 14, where as noted in the target article the violinist uses more ornamentation than in Duos 12 and 13. 
Table 3. Summary mean vector calculations for the phase relationship between violin and viola onsets, Duos 12-14.

\begin{tabular}{|l|l|l|l|}
\hline Variable & Duo12 & Duo13 & Duo14 \\
\hline Number of Observations & 21 & 20 & 19 \\
\hline Mean Vector $(\mu)$ & $326.0^{\circ}$ & $330.5^{\circ}$ & $338.0^{\circ}$ \\
\hline Length of Mean Vector $(r)$ & 0.921 & 0.938 & 0.906 \\
\hline
\end{tabular}

If the viola is on average about $22-34^{\circ}$ behind the violin, is this pattern the same for both $\mathrm{S}$ and $\mathrm{L}$ beat onsets? The phase diagrams for these three recordings show some differences, again, between Duos 12 and 13 on the one hand and Duo 14 on the other - in other words, the 'artificial' versions as against the 'normal', ornamented version (Figure 3). Within the viola part, the phase of the $\mathrm{L}$ onset with respect to the S-S period is respectively $147.6^{\circ}, 147.2^{\circ}$ and $148.1^{\circ}$ (indicated on the phase diagrams in Figure 3 with purple ticks). The violin onsets on the Short beats are respectively $29.6^{\circ}, 28.7^{\circ}$ and $5.3^{\circ}$ ahead of the viola: in performance Duo 14, therefore, the violinist takes a different approach to the other two versions of this tune, playing on average quite close to the viola's Short onsets. The violin onsets on the Long beats, however, are respectively $43.8^{\circ}, 33.5^{\circ}$ and $34.4^{\circ}$ ahead of the viola. In brief, whereas in Duos 12 and 13 the violinist consistently plays between $28.7^{\circ}$ and $43.8^{\circ}$ ahead of the violist, in Duo 14 he falls only $5.3^{\circ}$ ahead on the Short beats in contrast to his average position of $34.4^{\circ}$ ahead on the Longs. This could be regarded as an instance of 'soft entrainment' (Yoshida et al 2002), in which the coordination between the parts varies periodically: it is only evident in Duo 14, however. It also accounts for the unusually low figures calculated for the S:L ratio in the violin part (see Table 2): the L falls very early but the S does not, which means that in the violin part the $\mathrm{S}$ beat is shorter than in the other examples or in the viola part.

These charts may also provide an interpretation for the difference noted by the authors between 'small' and 'large' asynchronies. In Duo 12 on the Short beats, for instance, the phase angles seem to cluster with a group of 3 onsets falling close to $0^{\circ}$ and the rest closer to $45^{\circ}$ early $\left(315^{\circ}\right)$. A similar pattern may be noted for Duo 13's Shorts, but not for the Long beats or for Duo 14. Although the evidence is thin, this is consistent with the idea that in these two recordings the violinist either plays roughly in time with the violist (small asynchrony), or significantly early (large asynchrony). 

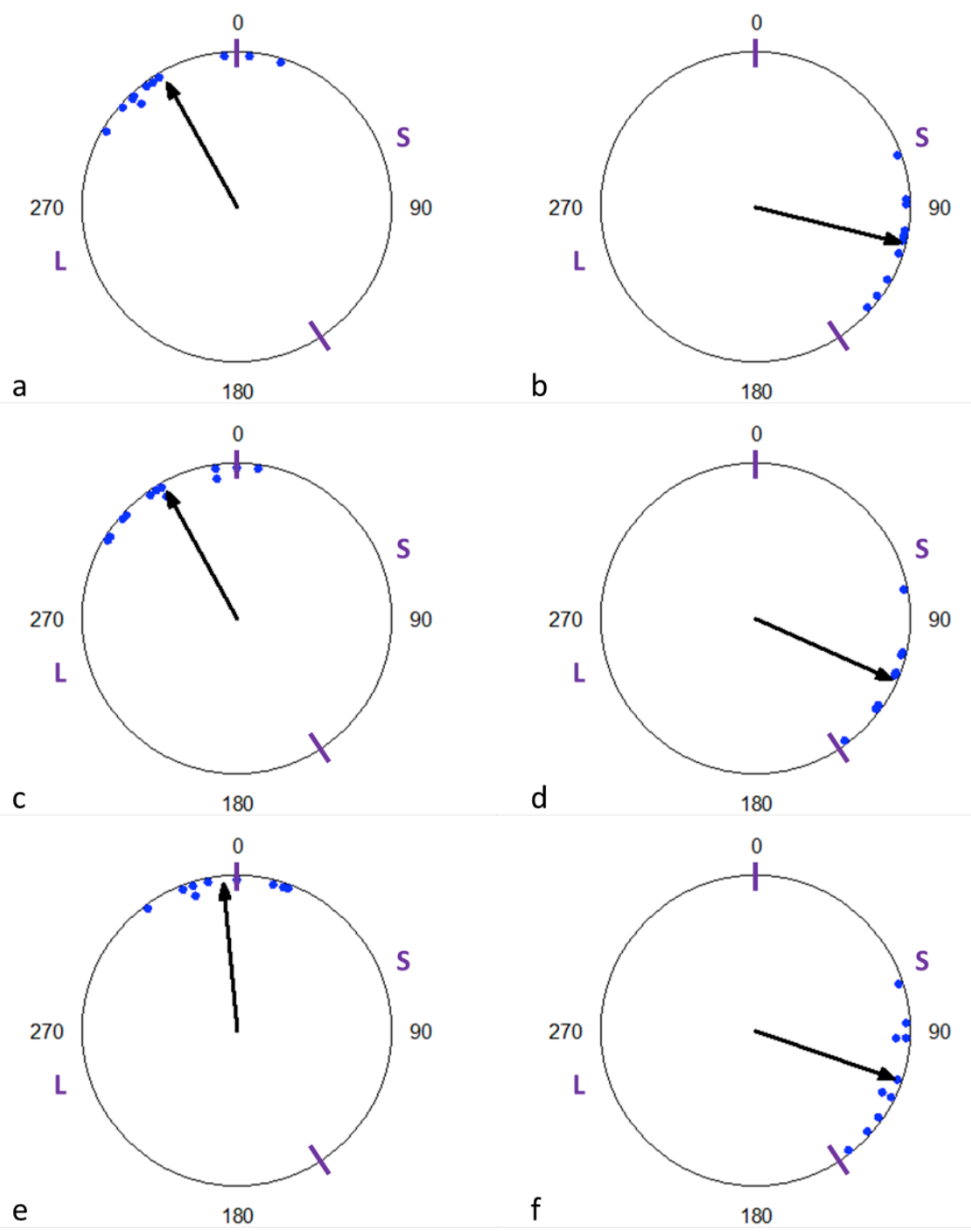

Figure 3. Phase diagrams for $\mathrm{S}$ and $\mathrm{L}$ beats for Duos 12-14. Purple ticks indicate the mean locations of the viola beats; blue circles indicate individual violin onsets, black arrows the mean vectors. (a) Duo 12 Short violin vs viola. $\mu=330.4^{\circ}, r=0.921$ (b) Duo 12 Long violin vs viola. $\mu=103.8^{\circ}, r=0.952$. (c) Duo 13 Short violin vs viola. $\mu=331.3^{\circ}, r=0.932$ (d) Duo 13 Long violin vs viola. $\mu=113.8^{\circ}, r=0.952$ (e) Duo 14 Short violin vs viola. $\mu=354.7^{\circ}, r=0.949$ (f) Duo 14 Long violin vs viola. $\mu=109.0^{\circ}, r=0.936$.

\section{SUMMARY}

My response has focused on two complementary aspects of the performance of this music, the S:L ratio and entrainment between the musicians. In the former case, my findings are as follows:

i. S:L ratios in this data set are found to be well spread over a continuum between (but not close to) $1: 1$ and $1: 2$, with a mean closer to $1: \sqrt{ } 2$ than to either $2: 3$ or $3: 4$. This adds weight to the argument (supported by Bonini Baraldi et al) that in some repertoires aksak rhythms are based not on a fast isochronous pulse, but rather on two distinct interval classes whose proportion is not close to a small integer ratio. There is however evidence that an attractor ratio at 2:3 may play a role in some examples: this may correspond to occasions when the beat is evenly subdivided, a hypothesis that remains to be tested.

ii. The distribution of ratios is different whether they are calculated for $\mathrm{S}+\mathrm{L}$ or $\mathrm{L}+\mathrm{S}$ periods (i.e. whether the Long follows or precedes the Short). Asymmetries are also found if coefficients of 
variation are computed for the period both ways around (although in this case the difference is small), and if correlations between S:L ratio and tempo are computed. These findings suggest that this factor should be considered in future studies: it may shed light, for instance, on whether or not the timing of periods of two or more intervals is controlled as such (hierarchically), and if so where the boundaries of the periods lie.

iii. There is a significant positive correlation between the S:L ratio and tempo: as tempo increases the ratio tends towards 1 , while at slower tempi the difference between Shorts and Longs is exaggerated. This factor is also worth considering in future analyses on nonisochronous rhythm.

The analysis of entrainment I have presented above suggests that this approach is a useful complement to asynchrony analysis. Over the three Duo recordings for which we have data, the violin onsets fall on average $22-34^{\circ}$ before the viola's: the fact that the viola's timing is much less variable than the violin's suggests that the viola provides a stable reference for the violinist to play around (the target article tends to the opposite interpretation, that the viola waits for the violin). More detailed entrainment analysis indicates a difference between Duo 14 and Duos 12 and 13 (the same tune played in a less typical style, with less ornamentation). In Duo 14, asynchronies on the $\mathrm{S}$ beat are relatively small because the violinist tends to play very close to the metrical position articulated by the violist, whereas on the L beat the violin plays quite early with respect to the viola and thus asynchronies tend to be larger - this alternation between more and less closely aligned beats is an example of soft entrainment. The suggestion in the target article that there may be a categorical difference between small and large asynchronies between violin and viola is, in this interpretation, a reflection of the fact that the violinist sometimes plays roughly on the viola's beat, but more often plays rather earlier.

I am not aware of any other studies of performed aksak rhythms that go into anything approaching the detail of this case study, or any that study entrainment in aksak contexts. I believe that the findings both those of the target article and those of my response - point to the value of such an approach, and hope that more will be attempted in the future. Overall, this case study points to the considerable potential for further empirical studies in this and related genres to contribute to both metrical theory and understanding of entrainment and interaction.

\section{ACKNOWLEDGEMENTS}

I would like to thank Filippo Bonini Baraldi for kindly making his original data available to me, and Tuomas Eerola, Peter Keller and Eric Clarke for their valuable advice.

\section{NOTES}

[1] Polak and London suggest in their study of Mande drumming that even where the proportions approximate to these small integer ratios, they "cannot be based on an isochronous substrate... as the IOIs for such pulses would simply be too short, exceeding the $100 \mathrm{~ms}$ limit for rhythmically salient events" (2014: [92]).

[2] Repp et al's conclusions speak to the issue of small-integer attractor ratios more generally, when they point out that "the attractor ratio... is not necessarily the mathematically simplest ratio... the fact that there is any consistent shift [in this study] is problematic for any theory of rhythmic perception and production which privileges simple, low order interval ratios." (2012: 221).

[3] See Arom 2004 for a more recent statement of his theory.

[4] London writes that "The 1:1 and 1:2 ratios stake out limiting conditions on S-L relationships for beats in NI meters." (2012: 135). The greater part of his discussion, however, is concerned with cases where these beat classes are subdivided and therefore $S: L$ is a ratio of integers, usually in the form $n: n+1$.

[5] This is also the geometrical mean of 2:3 and 3:4.

[6] In a couple of cases the means calculated in Excel given here are slightly different from those given in the original article. 
[7] It is worth noting that the S:L ratio for the longer CM recording (average 0.656) is lower than that for any of the 30 -second motion capture recordings. It is possible that the longer, more naturalistic performances, would produce a different overall picture.

[8] In contrast, Collier and Wright's experimental study shows a 2:3 ratio to be stable under acceleration (1995). In this case participants were expert Western musicians, and may have used subdivision to stabilize the intervals.

\section{REFERENCES}

Arom, S. (2004). L'aksak: Principes et typologie. Cahiers de musiques traditionnelles, 17, 11-48.

Benadon, F. (2006). Slicing the beat: jazz eighth-notes as expressive microrhythm. Ethnomusicology, 50(1), 73-98.

Blom, J-P. (1978). Principles of rhythmic structures in Balkan folk music. Antropologiska Studier, 25/26, $2-11$.

Bouët, J. (1997). Pulsations retrouvées: Les outils de la réalisation rythmique avant l'ère du métronome. Cahiers de Musiques Traditionnelles, 10, 107-125.

Clayton, M. (forthcoming) Non-isochronous metres in theory and in performance: The case of the North Indian rupak tal. In H. Neuhoff \& R. Polak, eds, Musical Metre in Comparative Perspective.

Clayton, M., Sager, R., \& Will, U. (2005). In time with the music: the concept of entrainment and its significance for ethnomusicology. European Meetings in Ethnomusicology, 11 (ESEM Counterpoint, 1), 375.

Collier, G. L., \& Wright, C. E. (1995). Temporal rescaling of simple and complex ratios in rhythmic tapping. Journal of Experimental Psychology: Human Perception and Performance, 21(3), 602-627.

Fraisse, P. (1978). Time and rhythm perception. In E. C. Carterette \& M. P. Friedman (Eds.), Handbook of Perception (Vol. 8, pp. 203-254). New York: Academic Press.

Jankowsky, R. C. (2013). Rhythmic elasticity, metric ambiguity, and ritual teleology in Tunisian Stambeli. Analytical Approaches to World Music, 3(1).

Kvifte, T. (2007). Categories and timing: on the perception of meter. Ethnomusicology, 51(1), 64-84.

London, J. (2012). Hearing in Time: Psychological Aspects of Musical Meter. New York: Oxford University Press. Second Edition.

Polak, R. \& London, J. (2014). Timing and meter in Mande drumming from Mali. Music Theory Online, $20(1)$.

Repp, B. H., London, J. \& Keller, P. E. (2012). Distortions in reproduction of two-interval rhythms: when the "attractor ratio" is not exactly 1:2. Music Perception, 30(2), 205-223.

Semjen, A., \& Ivry, R. B. (2001). The coupled oscillator model of between-hand coordination in alternatehand tapping: a reappraisal. Journal of Experimental Psychology: Human Perception and Performance, 27, $251-265$.

Singer, A. (1974). The metrical structure of Macedonian dance. Ethnomusicology, 18, 379-404.

Yoshida, T., Takeda, S. \& Yamamoto, S. (2002). The application of entrainment to musical ensembles. ICMAI 2002. 